\title{
Dynamics of entanglement between two harmonic modes in stable and unstable regimes
}

\author{
L. Rebón, N. Canosa, R. Rossignoli \\ Departamento de Física-IFLP, Universidad Nacional de La Plata, C.C. 67, 1900 La Plata, Argentina
}

\begin{abstract}
The exact dynamics of the entanglement between two harmonic modes generated by an angular momentum coupling is examined. Such system arises when considering a particle in a rotating anisotropic harmonic trap or a charged particle in a fixed harmonic potential in a magnetic field, and exhibits a rich dynamical structure, with stable, unstable and critical regimes according to the values of the rotational frequency or field and trap parameters. Consequently, it is shown that the entanglement generated from an initially separable gaussian state can exhibit quite distinct evolutions, ranging from quasiperiodic behavior in stable sectors to different types of unbounded increase in critical and unstable regions. The latter lead respectively to a logarithmic and linear growth of the entanglement entropy with time. It is also shown that entanglement can be controlled by tuning the frequency, such that it can be increased, kept constant or returned to a vanishing value just with stepwise frequency variations. Exact asymptotic expressions for the entanglement entropy in the different dynamical regimes are provided.
\end{abstract}

PACS numbers: 03.67.Bg,03.65.Ud,05.30.Jp

\section{INTRODUCTION}

The investigation of entanglement dynamics and growth in different physical systems is of great current interest [13]. Quantum entanglement is well known to be an essential resource for quantum teleportation [4] and pure state based quantum computation [5], where its increase with system size is necessary to achieve an exponential speedup over classical computation 6, 7]. And a large entanglement growth with time after starting from a separable state indicates that the system dynamics cannot be simulated efficiently by classical means $\mathbb{8}$, turning it suitable for quantum simulations.

The aim of this work is to examine the dynamics of the entanglement between two harmonic modes generated by an angular momentum coupling, and its ability to reproduce typical regimes of entanglement growth in more complex many body systems, when starting from an initial separable gaussian state. The latter can be chosen, for instance, as the ground state of the non-interacting part of the Hamiltonian, thus reproducing the typical quantum quench scenario [1, 2, 8]. The present system can be physically realized by means of a charged particle in a uniform magnetic field within a harmonic potential or by a particle confined in a rotating harmonic trap 9 12], where the field or rotational frequency provides an easily controllable coupling strength. Accordingly, it has been widely used in quite different physical contexts, such as rotating nuclei [1], 12], quantum dots in a magnetic field [13] and fast rotating Bose-Einstein condensates within the lowest Landau level approximation 14 19]. In spite of its simplicity, the model is able to exhibit a rich dynamical structure 20], with both stable and distinct types of unstable regimes, characterized by bounded as well as unbounded dynamics, when considering all possible values of the field or frequency in a general anisotropic potential. Nonetheless, being a quadratic Hamiltonian in the pertinent coordinates and momenta, the dynamics can be determined analytically in all regimes, and the entanglement between modes can be evaluated exactly through the gaussian state formalism 21 25]. For the same reason, the Hamiltonian is also suitable for simulation with optical techniques 26.

The main result we will show here is that due its nontrivial dynamical properties, the entanglement dynamics in the previous model can exhibit distinct regimes, including a quasiperiodic evolution in dynamically stable sectors, different types of logarithmic growth at the border between stable and unstable sectors (critical regime) and a linear increase in dynamically unstable sectors. The model is then able to mimic the three typical regimes for the entanglement growth with time after a quantum quench, arising in spin $1 / 2$ chains with Ising type couplings, according to the results of refs. 1, 8], which show a linear growth for short range couplings, a logarithmic growth for long range interactions and an oscillatory behavior for nearly infinite range interactions, when considering a half-chain bipartition. We also mention that the static ground state entanglement of the present model also exhibits critical behavior at the border of instability 27. Mode entanglement dynamics in related harmonic models within stable regimes were previously studied in 28 30], while critical behavior and entanglement in ultrastrong-coupled oscillators (through a different interaction) were considered in 31]. Other relevant aspects of entanglement dynamics and generation in spin systems were discussed in 32 35.

In sec. II we discuss the exact dynamics of the system and describe the different regimes arising for strong coupling in anisotropic potentials. The entanglement evolution in gaussian states is then examined in detail in sec. III including its exact evaluation through the covariance matrix formalism and the exact asymptotic behavior in the distinct dynamical regimes. Explicit results, including the possibility of entanglement control through 
a stepwise varying frequency, are also shown. Conclusions are finally provided in IV

\section{MODEL AND EXACT DYNAMICS}

\section{A. Hamiltonian}

We consider two harmonic systems with coordinates and momenta $Q_{\mu}, P_{\mu}, \mu=x, y$, coupled through their angular momentum $L_{z}=Q_{x} P_{y}-Q_{y} P_{x}$. The Hamiltonian is

$$
\begin{aligned}
H & =H_{0}-\Omega L_{z}, \\
H_{0} & =\frac{P_{x}^{2}+P_{y}^{2}}{2 m}+\frac{1}{2}\left(K_{x} Q_{x}^{2}+K_{y} Q_{y}^{2}\right) .
\end{aligned}
$$

Eq. (11) describes, for instance, the motion in the $x, y$ plane of a particle of charge $e$ and mass $m$ within a harmonic trap of spring constants $\tilde{K}_{\mu}$ in a uniform field $\boldsymbol{H}$ along the $z$ axis 11, 12], if $\Omega=\frac{e|\boldsymbol{H}|}{2 m c}$ stands for half the cyclotron frequency and $K_{\mu}=\tilde{K}_{\mu}+m \Omega^{2}$.

It also determines the intrinsic motion of a particle in a harmonic trap with constants $K_{\mu}$ which rotates around the $z$ axis with frequency $\Omega$. In this case 11, 12, the actual Hamiltonian is $H(t)=R(t) H_{0} R^{\dagger}(t)$, with $R(t)=$ $e^{-i \Omega L_{z} t / \hbar}$ the rotation operator, but averages of rotating observables $O(t)=R(t) O R^{\dagger}(t)$ evolve like those of $O$ under the time-independent "cranked" Hamiltonian (1).

Replacing $Q_{\mu}=q_{\mu} / \sqrt{m \Omega_{0} / \hbar}, P_{\mu}=p_{\mu} \sqrt{\hbar m \Omega_{0}}$, with $q_{\mu}, p_{\mu}$ dimensionless coordinates and momenta $\left(\left[q_{\mu}, p_{\nu}\right]=i \delta_{\mu \nu},\left[q_{\mu}, q_{\nu}\right]=\left[p_{\mu}, p_{\nu}\right]=0\right)$ and $\Omega_{0}$ a reference frequency, we have $H=\hbar \Omega_{0} h$, with

$$
\begin{aligned}
& h=h_{0}-\omega l_{z}, \quad h_{0}=\frac{1}{2}\left(p_{x}^{2}+p_{y}^{2}+k_{x} q_{x}^{2}+k_{y} q_{y}^{2}\right), \\
& l_{z}=q_{x} p_{y}-q_{y} p_{x}=-i\left(b_{x}^{\dagger} b_{y}-b_{y}^{\dagger} b_{x}\right),
\end{aligned}
$$

where $k_{\mu}=K_{\mu} /\left(m \Omega_{0}^{2}\right)$ and $\omega=\Omega / \Omega_{0}$ are dimensionless $\left(\Omega_{0}\right.$ can be used to set $\left.\left|k_{x}\right|=1\right)$ and $b_{\mu}=\frac{q_{\mu}+i p_{\mu}}{\sqrt{2}}$ are the boson annihilation operators associated with $q_{\mu}, p_{\mu}$. The $l_{z}$ coupling (4) is then seen to conserve the associated total boson number $N=\sum_{\mu=x, y} b_{\mu}^{\dagger} b_{\mu}$, being in fact the same as that describing the mixing of two modes of radiation field passing through a beam splitter [5]. Notice, however, that $\left[h_{0}, N\right] \neq 0$ unless $k_{x}=k_{y}=1$ (stable isotropic trap).

\section{B. Exact evolution}

The Heisenberg equations of motion $i d o / d t=-[h, o]$ for the operators $q_{\mu}, p_{\mu}$ (with $t=\Omega_{0} T$ and $T$ the actual time) become

$$
\begin{array}{ll}
\frac{d q_{x}}{d t}=p_{x}+\omega q_{y}, & \frac{d q_{y}}{d t}=p_{y}-\omega q_{x} \\
\frac{d p_{x}}{d t}=-k_{x} q_{x}+\omega p_{y}, & \frac{d p_{y}}{d t}=-k_{y} q_{y}-\omega p_{x}
\end{array},
$$

and can be written in matrix form as

$$
\begin{aligned}
i \frac{d}{d t} \mathcal{O} & =\mathcal{H O}, \\
\mathcal{O} & =\left(\begin{array}{l}
q_{x} \\
q_{y} \\
p_{x} \\
p_{y}
\end{array}\right), \mathcal{H}=i\left(\begin{array}{cccc}
0 & \omega & 1 & 0 \\
-\omega & 0 & 0 & 1 \\
-k_{x} & 0 & 0 & \omega \\
0 & -k_{y} & -\omega & 0
\end{array}\right) .
\end{aligned}
$$

The system dynamics is then fully determined by the matrix $\mathcal{H}$. We may write the general solution of (6) as

$$
\mathcal{O}(t)=\mathcal{U}(t) \mathcal{O}, \quad \mathcal{U}(t)=\exp [-i \mathcal{H} t],
$$

where $\mathcal{O} \equiv \mathcal{O}(0)$.

In spite of their simplicity, Eqs.(5) can lead to quite distinct dynamical regimes according to the values of $\omega$ and $k_{\mu}$, as the eigenvalues of $\mathcal{H}$, which is in general a non-hermitian matrix, can become imaginary or complex away from stable regions 20]. Moreover, $\mathcal{H}$ can also become non-diagonalizable at the boundaries between distinct regimes, exhibiting non-trivial Jordan canonical forms 20]. Nonetheless, as

$$
\mathcal{H}^{2}=\left(\begin{array}{cccc}
k_{x}+\omega^{2} & 0 & 0 & -2 \omega \\
0 & k_{y}+\omega^{2} & 2 \omega & 0 \\
0 & \omega\left(k_{x}+k_{y}\right) & k_{x}+\omega^{2} & 0 \\
-\omega\left(k_{x}+k_{y}\right) & 0 & 0 & k_{y}+\omega^{2}
\end{array}\right),
$$

the eigenvalues of $\mathcal{H}$ are determined by $2 \times 2$ blocks, and given by $\lambda_{ \pm}$and $-\lambda_{ \pm}$, with

$$
\lambda_{ \pm}=\sqrt{\varepsilon_{+}+\omega^{2} \pm \Delta},
$$

where $\varepsilon_{ \pm}=\frac{k_{x} \pm k_{y}}{2}$ and $\Delta=\sqrt{\varepsilon_{-}^{2}+4 \omega^{2} \varepsilon_{+}}$.

We can then write the solution (8) explicitly as

$$
\left(\begin{array}{c}
q_{x}(t) \\
q_{y}(t) \\
p_{x}(t) \\
p_{y}(t)
\end{array}\right)=\left(\begin{array}{cccc}
u_{x x} & u_{x y} & v_{x x} & v_{x y} \\
u_{y x} & u_{y y} & -v_{x y} & v_{y y} \\
w_{x x} & w_{x y} & u_{x x} & -u_{y x} \\
-w_{x y} & w_{y y} & -u_{x y} & u_{y y}
\end{array}\right)\left(\begin{array}{c}
q_{x} \\
q_{y} \\
p_{x} \\
p_{y}
\end{array}\right)
$$

where

$$
\begin{aligned}
u_{y x}^{x x} & =\frac{\left(\Delta \pm \varepsilon_{-}\right) \cos \lambda_{+} t+\left(\Delta \mp \varepsilon_{-}\right) \cos \lambda_{-} t}{2 \Delta}, \\
u_{y y} & = \pm \omega \frac{\left(\Delta \mp \varepsilon_{-}+2 \varepsilon_{+}\right) \frac{\sin \lambda_{+} t}{\lambda_{+}}+\left(\Delta \pm \varepsilon_{-}-2 \varepsilon_{+}\right) \frac{\sin \lambda_{-}}{\lambda_{-}}}{2 \Delta} \\
v_{x x}^{x} & =\frac{\left(\Delta \pm \varepsilon_{-}+2 \omega^{2}\right) \frac{\sin \lambda_{+} t}{\lambda_{+}}+\left(\Delta \mp \varepsilon_{-}-2 \omega^{2}\right) \frac{\sin \lambda_{-} t}{\lambda_{-}}}{2 \Delta}, \\
v_{x y} & =\frac{\omega\left(-\cos \lambda_{+} t+\cos \lambda_{-} t\right)}{\Delta}, w_{x y}=-\varepsilon_{+} v_{x y}, \\
w_{x y}^{x y} & =\frac{-\left(\Delta \pm \varepsilon_{-}\right)\left(\Delta \pm \varepsilon_{-}+2 \varepsilon_{+}\right) \frac{\sin \lambda_{+} t}{\lambda_{+}}+\left(\Delta \mp \varepsilon_{-}\right)\left(\Delta \mp \varepsilon_{-}-2 \varepsilon_{+}\right) \frac{\sin \lambda_{-} t}{4 \Delta}}{\lambda_{-}} .
\end{aligned}
$$

The matrix $\mathcal{U}(t)$ is real for any real values of $\omega, k_{\mu}$ and $t$, including unstable regimes where $\Delta$ and/or $\lambda_{ \pm}$can be imaginary or complex 20. It represents always a linear canonical transformation of the $q_{\mu}, p_{\mu}$, satisfying

$$
\mathcal{U}(t) \mathcal{M U}^{t}(t)=\mathcal{M}, \quad \mathcal{M}=i\left(\begin{array}{cc}
0 & I \\
-I & 0
\end{array}\right),
$$


( $I$ denotes the $2 \times 2$ identity matrix) which ensures the preservation of commutation relations $\left(\left[\mathcal{O}_{i}, \mathcal{O}_{j}\right]=\mathcal{M}_{i j}\right)$. It corresponds to a proper Bogoliubov transformation of the associated boson operators.

For $\omega=0$, we recover from Eqs. (11)-112) the decoupled harmonic evolution $q_{\mu}(t)=q_{\mu} \cos \omega_{\mu} t+$ $\omega_{\mu}^{-1} p_{\mu} \sin \omega_{\mu} t, p_{\mu}(t)=p_{\mu} \cos \omega_{\mu} t-q_{\mu} \omega_{\mu} \sin \omega_{\mu} t$, where $\omega_{\mu}=\sqrt{k_{\mu}}$ for $\mu=x, y$. Off-diagonal terms $u_{x y}, u_{y x}$, $v_{x y}, w_{x y}$ in (12) are $O(\omega)$ for small $\omega$.

On the other hand, in the isotropic case $k_{x}=k_{y}=k$ (where $\Delta=2 \omega \sqrt{k}$ and $\left|\lambda_{ \pm}\right|=|\sqrt{k} \pm \omega|$ ), $\left[l_{z}, h\right]=0$ and the evolution provided by Eqs. (11)-(12) is just the rotation of identical single mode evolutions:

$$
\begin{aligned}
\mathcal{U}(t) & =\exp \left[i \omega \mathcal{L}_{z} t\right] \exp \left[-i \mathcal{H}_{0} t\right] \\
\exp \left[i \omega \mathcal{L}_{z} t\right] & =\left(\begin{array}{cc}
R^{\dagger}(t) & 0 \\
0 & R^{\dagger}(t)
\end{array}\right), R^{\dagger}(t)=\left(\begin{array}{cc}
\cos \omega t & \sin \omega t \\
-\sin \omega t & \cos \omega t
\end{array}\right)
\end{aligned}
$$

In particular, the Landau case (free particle in a magnetic field) corresponds to $k_{x}=k_{y}=\omega^{2}$, where $\lambda_{+}=2 \omega$ and $\lambda_{-}=0$.

\section{Dynamical regimes}

The distinct dynamical regimes exhibited by this system for $\omega \neq 0$ are summarized in Fig. 1 Let us first consider the standard stable case $k_{x}>0, k_{y}>0$ (first quadrant). The eigenvalues $\lambda_{ \pm}$are here both real and non-zero in sectors $\mathbf{A}$ and $\mathbf{B}$, defined by

$$
\begin{array}{ll}
\omega^{2}<\operatorname{Min}\left[k_{x}, k_{y}\right] & (\text { sector } \mathbf{A}), \\
\omega^{2}>\operatorname{Max}\left[k_{x}, k_{y}\right] & (\text { sector } \mathbf{B}),
\end{array}
$$

when $k_{x}>0, k_{y}>0 . \mathbf{A}$ is the full stable sector where $h$ is positive definite, whereas $\mathbf{B}$ is that where the system, though unstable, remains dynamically stable 20 (see also Appendix). If $\omega^{2}$ lies between these values (sector $\mathbf{D}), \lambda_{-}$becomes imaginary (with $\lambda_{+}$remaining real), leading to a frequency window where the system becomes dynamically unstable (unbounded motion), with $\sin \left(\lambda_{-} t\right) / \lambda_{-}=\sinh \left(\left|\lambda_{-}\right| t\right) /\left|\lambda_{-}\right|$in Eqs. (12).

At the border between $\mathbf{D}$ and $\mathbf{A}$ or $\mathbf{B}\left(\omega^{2}=k_{x}\right.$ or $\left.\omega^{2}=k_{y}\right), \lambda_{-}=0$ (with $\lambda_{+}>0$ ) and $\mathcal{H}$ becomes nondiagonalizable if $k_{y} \neq k_{x}$, although $\mathcal{H}^{2}$ remains diagonalizable. The system becomes here equivalent to a stable oscillator plus a free particle [20] (see Appendix), and we should just replace $\sin \left(\lambda_{-} t\right) / \lambda_{-}$by its limit $t$ in Eqs. (12), which leads again to an unbounded motion.

Considering now the possibility of unstable potentials ( $k_{x}<0$ and/or $k_{y}<0$, remaining quadrants), the dynamically stable sector $\mathbf{B}$ extends into this region provided $k_{x}>0>k_{y}>-3 k_{x}$ (or viceversa) and

$$
\operatorname{Max}\left[k_{x}, k_{y}\right]<\omega^{2}<-\varepsilon_{-}^{2} /\left(4 \varepsilon_{+}\right),
$$

where the upper bound applies only when $\varepsilon_{+}<0$ (i.e., $-3 k_{x}<k_{y}<-k_{x}$ or viceversa). Eq. (17) defines a frequency window where the unstable system becomes

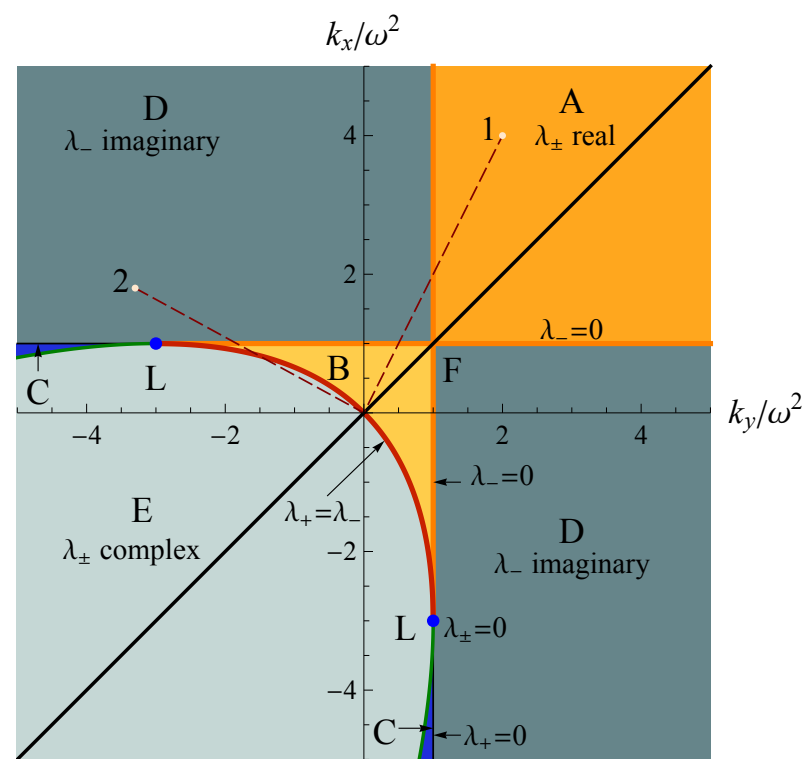

FIG. 1. Dynamical phase diagram of the system described by Hamiltonian (1). The evolution of the operators $q_{\mu}, p_{\mu}$ is quasiperiodic in the dynamically stable sectors $\mathbf{A}, \mathbf{B}$, where the eigenfrequencies $\lambda_{ \pm}$are both real, but unbounded in the remaining sectors, with $\lambda_{-}$imaginary in $\mathbf{D}$, both $\lambda_{ \pm}$imaginary in $\mathbf{C}$, and $\lambda_{ \pm}$complex conjugates in $\mathbf{E}$. At the borders between these regions (except from the Landau point $F$ ) the matrix $\mathcal{H}$ is non-diagonalizable and the evolution is also unbounded, with $\lambda_{-}=0$ at the borders between $\mathbf{D}$ and $\mathbf{A}$ or $\mathbf{B}, \lambda_{+}=0$ at the border between $\mathbf{D}$ and $\mathbf{C}, \lambda_{+}=\lambda_{-}$at the curve separating $\mathbf{E}$ from $\mathbf{B}$ and $\mathbf{C}$ and $\lambda_{ \pm}=0$ at the critical points $L$. Dashed lines indicate the path described as $\omega$ is increased at fixed $k_{\mu}$, showing that the system dynamics will become unbounded (bounded) in a certain frequency window when starting at $1(2)$. The black line indicates the isotropic case $k_{x}=k_{y}$, where entanglement will be periodic (see sec. III).

dynamically stable $\left(\lambda_{ \pm}\right.$real). Beyond this sector, either $\lambda_{-}$becomes imaginary (sectors $\mathbf{D}$ ) or both $\lambda_{ \pm}$become imaginary (sectors $\mathbf{C}$ ) or complex conjugates (sector $\mathbf{E}$, where $\Delta$ is imaginary), and the dynamics becomes again unbounded. This is also the case at the borders between $\mathbf{D}$ and $\mathbf{B}\left(\lambda_{+}>0, \lambda_{-}=0\right)$ and also $\mathbf{D}$ and $\mathbf{C}\left(\lambda_{+}=0\right.$, $\lambda_{-}$imaginary) where $\mathcal{H}$ is non-diagonalizable (see Appendix for more details).

The critical curve $\Delta=0$, i.e.,

$$
\omega^{2}=-\varepsilon_{-}^{2} /\left(4 \varepsilon_{+}\right)
$$

where $\varepsilon_{+}<0$, separates sectors $\mathbf{B}$ and $\mathbf{C}$ from $\mathbf{E}$ and deserves special attention. At this curve, $\lambda_{ \pm}=$ $\lambda=\sqrt{\varepsilon_{+}+\omega^{2}}$ and both $\mathcal{H}$ and $\mathcal{H}^{2}$ become nondiagonalizable, with $\lambda$ real at the border between $\mathbf{B}$ and $\mathbf{E}$ and imaginary at that between $\mathbf{C}$ and $\mathbf{E}$. The evaluation of $\mathcal{U}(t)$ in Eq. (8) can in this case be obtained through the pertinent Jordan decomposition of $\mathcal{H}$ (two $2 \times 2$ blocks 20 ), but the final result coincides with the 
$\Delta \rightarrow 0$ limit of Eqs. (12). This leads to the elements

$$
\begin{aligned}
u_{x y}^{x} & =\cos \lambda t \mp t \varepsilon_{-} \frac{\sin \lambda t}{2 \lambda}, \\
u_{x y}^{x y} & = \pm \omega \frac{t \lambda\left(\varepsilon_{+} \mp \varepsilon_{-} / 2\right) \cos \lambda t+\left(\omega^{2} \pm \varepsilon_{-} / 2\right) \sin \lambda t}{\lambda^{3}}, \\
v_{y x}^{x x} & =\frac{t \lambda\left(\omega^{2} \pm \varepsilon_{-} / 2\right) \cos \lambda t+\left(\varepsilon_{+} \mp \varepsilon_{-} / 2\right) \sin \lambda t}{\lambda^{3}}, \\
v_{x y} & =\omega t \frac{\sin \lambda t}{\lambda}, w_{x y}=-\varepsilon_{+} v_{x y}, \\
w_{\substack{x y \\
y y}} & =\frac{\varepsilon_{+} t \lambda\left(\omega^{2} \mp \varepsilon_{-} / 2\right) \cos \lambda t-\left(\varepsilon_{+}+2 \omega^{2}\right)\left(\varepsilon_{+} \pm \varepsilon_{-} / 2\right) \sin \lambda t}{\lambda^{3}},
\end{aligned}
$$

which contain terms proportional to $t$. The evolution is, therefore, always unbounded along this curve.

Finally, if both $\Delta$ and $\lambda=\sqrt{\varepsilon_{+}+\omega^{2}}$ vanish, which occurs when $\varepsilon_{+}=-\omega^{2}=-\left|\varepsilon_{-}\right| / 2$, i.e.,

$$
\omega^{2}=k_{x}=-k_{y} / 3 \text {, }
$$

(or $\omega^{2}=k_{y}=-k_{x} / 3$ ), the system exhibits a remarkable critical point (points $L$ in Fig. 1), where $\lambda_{ \pm}=0$ and sectors $\mathbf{B}, \mathbf{C}, \mathbf{D}$ and $\mathbf{E}$ meet. Here both $\mathcal{H}$ and $\mathcal{H}^{2}$ are non-diagonalizable, with $\mathcal{H}$ represented by a single $4 \times 4$ Jordan Block (inseparable pair 20]). By using this form or taking the $\lambda \rightarrow 0$ limit in Eqs. (19), we obtain in this case a purely polynomial (and hence also unbounded) evolution, involving terms up to the third power of $t$ : The elements of $\mathcal{U}(t)$ become

$$
\begin{aligned}
u_{x x} & =1 \mp \omega^{2} t^{2} & & \\
u_{x y} & =\omega t\left(1+\frac{2}{3} \omega^{2} t^{2}\right), & u_{y x} & =-\omega t, \\
v_{x x} & =t\left(1-\frac{2}{3} \omega^{2} t^{2}\right), & v_{y y} & =t, \\
v_{x y} & =\omega t^{2}, & & w_{x y}=\omega^{3} t^{2}, \\
w_{x x} & =-\omega^{2} t, & w_{y y} & =\omega^{2} t\left(3+\frac{2}{3} \omega^{2} t^{2}\right) .
\end{aligned}
$$

Nonetheless, we remark that Eq. (13) remains satisfied (in both cases (19) and (21)).

\section{DYNAMICS OF ENTANGLEMENT IN GAUSSIAN STATES}

\section{A. Exact evaluation}

Let us now consider the evolution of the entanglement between the $x$ and $y$ modes, starting from an initially separable pure gaussian state. Since the evolution is equivalent to the linear canonical transformation (8), the state will remain gaussian $\forall t$, which entails that entanglement will be completely determined by the pertinent covariance matrix 22, 23].

We may then assume that at $t=0,\left\langle q_{\mu}\right\rangle=\left\langle p_{\mu}\right\rangle=0$ for $\mu=x, y(\langle\mathcal{O}\rangle=0)$, such that these mean values will vanish $\forall t\left(\langle\mathcal{O}\rangle_{t}=\langle\mathcal{O}(t)\rangle=0\right.$, as implied by Eq. (11)). We may then define the covariance matrix as

$$
\begin{aligned}
\mathcal{C} & =\left\langle\mathcal{O O}^{t}\right\rangle-\frac{1}{2} \mathcal{M} \\
& =\left(\begin{array}{cccc}
\left\langle q_{x}^{2}\right\rangle & \left\langle q_{x} q_{y}\right\rangle & \frac{\left\langle q_{x} p_{x}+p_{x} q_{x}\right\rangle}{2} & \left\langle q_{x} p_{y}\right\rangle \\
\left\langle q_{x} q_{y}\right\rangle & \left\langle q_{y}^{2}\right\rangle & \left\langle q_{y} p_{x}\right\rangle & \frac{\left\langle q_{y} p_{y}+p_{y} q_{y}\right\rangle}{2} \\
\frac{\left\langle q_{x} p_{x}+p_{x} q_{x}\right\rangle}{2} & \left\langle q_{y} p_{x}\right\rangle & \left\langle p_{x}^{2}\right\rangle & \left\langle p_{x} p_{y}\right\rangle \\
\left\langle q_{x} p_{y}\right\rangle & \frac{\left\langle q_{y} p_{y}+p_{y} q_{y}\right\rangle}{2} & \left\langle p_{x} p_{y}\right\rangle & \left\langle p_{y}^{2}\right\rangle
\end{array}\right)
\end{aligned}
$$

which, according to Eqs. (8) and [13), will evolve as

$$
\mathcal{C}(t)=\mathcal{U}(t) \mathcal{C}(0) \mathcal{U}^{t}(t) .
$$

The entanglement between the two modes will now be determined by the symplectic eigenvalue $\tilde{f}(t)=f(t)+1 / 2$ of the single mode covariance matrix $\mathcal{C}_{\mu}(t)=\left\langle\mathcal{O}_{\mu} \mathcal{O}_{\mu}^{t}\right\rangle_{t}-$ $\frac{1}{2} \mathcal{M}$, submatrix of (23), where $\mathcal{O}_{\mu}=\left(q_{\mu}, p_{\mu}\right)^{t}$. Here $f(t)$ is a non-negative quantity representing the average boson occupation $\left\langle a_{\mu}^{\dagger}(t) a_{\mu}(t)\right\rangle$ of the mode $\left(a_{\mu}(t)\right.$ is the local boson operator satisfying $\left.\left\langle a_{\mu}^{2}(t)\right\rangle=0\right)$, which is the same for both modes $\left(f_{x}(t)=f_{y}(t)\right)$ when the global state is gaussian and pure. It is given by

$$
f(t)=\sqrt{\left\langle q_{\mu}^{2}\right\rangle_{t}\left\langle p_{\mu}^{2}\right\rangle_{t}-\left\langle q_{\mu} p_{\mu}+p_{\mu} q_{\mu}\right\rangle_{t}^{2} / 4}-\frac{1}{2} .
$$

Eq. (24) is just the deviation from minimum uncertainty of the mode, and can be directly determined from the elements of (23).

The von Neumann entanglement entropy between the two modes becomes

$$
\begin{aligned}
S(t) & =-\operatorname{Tr} \rho_{\mu}(t) \ln \rho_{\mu}(t) \\
& =-f(t) \ln f(t)+[1+f(t)] \ln [1+f(t)],
\end{aligned}
$$

where $\rho_{\mu}(t)$ denotes the reduced state of the mode. Eq. (25) is an increasing concave function of $f(t)$. For future use, we note that for large and small $f(t)$,

$$
\begin{aligned}
& S(t) \approx \ln f(t)+1+O\left(f^{-1}\right), \\
& S(t) \approx f(t)[-\ln f(t)+1]+O\left(f^{2}\right) .
\end{aligned}
$$

Other entanglement entropies, like the Renyi entropies $S_{\alpha}(t)=\frac{\ln \operatorname{Tr} \rho_{\mu}^{\alpha}(t)}{1-\alpha}, \alpha>0$, and the linear entropy $S_{2}(t)=$ $1-\operatorname{Tr} \rho_{\mu}^{2}(t)$ (of experimental interest as $\operatorname{Tr} \rho^{2}$ and in general $\operatorname{Tr} \rho^{n}$ can be measured without performing a full state tomography [3, 36]), are obviously also determined by $f(t)$, since $\operatorname{Tr} \rho_{\mu}^{\alpha}=\left[\left(1+f_{\mu}\right)^{\alpha}-f_{\mu}^{\alpha}\right]^{-1}(\alpha>0)$.

The initial covariance matrix $\mathcal{C}(0)$ will be here assumed of the form

$$
\mathcal{C}(0)=\frac{1}{2}\left(\begin{array}{cccc}
\alpha_{x}^{-1} & 0 & 0 & 0 \\
0 & \alpha_{y}^{-1} & 0 & 0 \\
0 & 0 & \alpha_{x} & 0 \\
0 & 0 & 0 & \alpha_{y}
\end{array}\right),
$$

where $\alpha_{\mu}=2\left\langle p_{\mu}^{2}\right\rangle$, such that $\alpha_{\mu}=\sqrt{k_{\mu}}$ if the system is initially in the separable ground state of $h_{0}$, as in the typical quantum quench scenario [1]. For fixed isotropic initial conditions we will just take $\alpha_{x}=\alpha_{y}=1$.

For these initial conditions, we first notice that for small $t$, Eqs. (12) and (24) yield

$$
f(t) \approx \frac{\left(\alpha_{x}-\alpha_{y}\right)^{2}}{4 \alpha_{x} \alpha_{y}}(\omega t)^{2}+O\left(t^{4}\right),
$$

which indicates a quadratic initial increase of $f(t)$ with time for any anisotropic initial covariance. Eq. (29) is 


\begin{tabular}{|c|c|c|}
\hline Sectors & Average occupation & Entanglement entropy \\
\hline A, B & Quasiperiodic & Quasiperiodic \\
\hline C & $f(t) \propto e^{\left(\left|\lambda_{-}\right|+\left|\lambda_{-}\right|\right) t}$ & $S(t) \approx\left(\left|\lambda_{-}\right|+\left|\lambda_{-}\right|\right) t$ \\
\hline D & $f(t) \propto e^{\left|\lambda_{-}\right| t}$ & $S(t) \approx\left|\lambda_{-}\right| t$ \\
\hline E & $f(t) \propto e^{2|\operatorname{Im}(\lambda)| t}$ & $S(t) \approx 2\left|\operatorname{Im}\left(\lambda_{ \pm}\right)\right| t$ \\
\hline Borders A-D, B-D & $f(t) \propto t$ & $S(t) \approx S_{0}(t)+\ln t$ \\
\hline Border B-E & $f(t) \propto t^{2}$ & $S(t) \approx S_{1}+2 \ln t$ \\
\hline Points L & $f(t) \propto t^{4}$ & $S(t) \approx S_{2}+4 \ln t$ \\
\hline Line $k_{x}=k_{y}$ & Periodic $\left(\alpha_{x} \neq \alpha_{y}\right)$ & Periodic $\left(\alpha_{x} \neq \alpha_{y}\right)$ \\
\hline
\end{tabular}

TABLE I. The asymptotic evolution of the average occupation (24) and the entanglement entropy (25) in the different dynamical sectors indicated in Fig. 11 Entanglement is bounded in the stable sectors $\mathbf{A}, \mathbf{B}$, but increases linearly (with $t$ ) in the unstable sectors $\mathbf{C}, \mathbf{D}, \mathbf{E}$, and logarithmically at the border between stable and unstable sectors, provided $k_{x} \neq k_{y}$. In the isotropic case $k_{x}=k_{y}=k$ it remains periodic for any value of $k$ and anisotropic initial conditions.

independent of the oscillator parameters $k_{\mu}$ and proportional to $\omega^{2}$. However, for isotropic initial conditions $\alpha_{x}=\alpha_{y}$, quadratic terms vanish and we obtain instead a quartic initial increase, driven by the oscillator anisotropy $\varepsilon_{-}$:

$$
f(t) \approx \frac{\varepsilon_{-}^{2} \omega^{2}}{4 \alpha_{x}^{2}} t^{4}+O\left(t^{6}\right)
$$

Eq. (27) implies a similar initial behavior (except for a factor $\ln t$ ) of the entanglement entropy.

Next, in the isotropic case $k_{x}=k_{y}=k$, the exact expression for $f(t)$ becomes quite simple, since the rotation is decoupled from the internal motion of the modes (Eq. (14)), and entanglement arises solely from rotation and initial anisotropy. We obtain

$$
f(t)=\frac{1}{2} \sqrt{1+\frac{\left(\alpha_{x}-\alpha_{y}\right)^{2}}{4 \alpha_{x} \alpha_{y}} \sin ^{2}(2 \omega t)}-\frac{1}{2} .
$$

Entanglement will then simply oscillate with frequency $4 \omega$ if $\alpha_{x} \neq \alpha_{y}$, being independent of the trap parameter $k$, since the latter affects just a local transformation decoupled from the rotation. Eq. (31) holds in fact even if $k$ becomes negative (unstable potential) or vanishes.

In the general case, the previous decoupling no longer holds and the explicit expression for $f(t)$ becomes quite long. The main point we want to show is that the different dynamical regimes lead to distinct behaviors of $f(t)$, and hence of the generated entanglement entropy $S(t)$, which are summarized in Table प. We now describe them in detail.

\section{B. Evolution in stable sectors}

In the dynamically stable sectors $\mathbf{A}$ and $\mathbf{B}$ of Fig. 1 both $\lambda_{ \pm}$are real and non-zero, implying that the evolu-

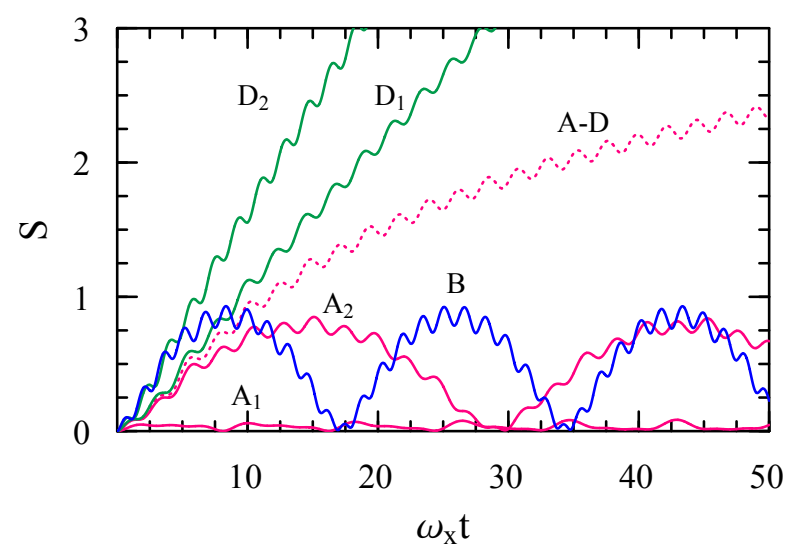

FIG. 2. The evolution of the entanglement entropy (25) between the two modes for $k_{y}=0.3 k_{x}>0$ and frequencies $\omega / \omega_{y}=0.5\left(\mathbf{A}_{1}\right), 0.95\left(\mathbf{A}_{2}\right), 1$ (A-D), $1.05\left(\mathbf{D}_{1}\right), 1.7\left(\mathbf{D}_{2}\right)$ and $1.95(\mathbf{B})$, where $\omega_{\mu}=\sqrt{k_{\mu}}$ and the label indicates the corresponding sector in Fig. 1 S $S(t)$ is quasiperiodic in curves $\mathbf{A}_{1}, \mathbf{A}_{2}$ and $\mathbf{B}$, but increases logarithmically (on average) in $\mathbf{A}-\mathbf{D}$, and linearly in $\mathbf{D}_{1}, \mathbf{D}_{2}$. The initial state is the separable ground state of $H_{0}$ (uncoupled oscillators).

tion of $f(t)$ and $S(t)$ will be quasiperiodic, as seen in Fig. 2 (curves $\mathbf{A}_{1}, \mathbf{A}_{2}$ and $\mathbf{B}$ ). The initial state was chosen as the ground state of $h_{0}\left(\alpha_{\mu}=\sqrt{k_{\mu}}\right.$ in (28) $)$. Starting from point 1 in sector A (Fig. 1), the generated entanglement $S(t)$ remains small when $\omega$ is well below the first critical value $\omega_{y}=\sqrt{k_{y}}$ (curve $\mathbf{A}_{1}$ ). As $\omega$ increases, $S(t)$ will exhibit increasingly higher maxima, showing a typical resonant behavior for $\omega$ close to $\omega_{y}$ (border with sector $\mathbf{D})$, where $\lambda_{-}$vanishes. Near this border, $S(t)$ will essentially exhibit large amplitude low frequency oscillations determined by $\lambda_{-}$, with maxima at $t \approx t_{m}=\frac{m \pi}{2 \lambda_{-}}$ ( $m$ odd), plus low amplitude high frequency oscillations determined by $\lambda_{+}$, as seen in curve $\mathbf{A}_{2}$.

As $\omega$ increases, the system enters dynamically unstable sectors for $\omega_{y} \leq \omega \leq \omega_{x}=\sqrt{k_{x}}$, and the evolution becomes unbounded (curves $\mathbf{A}-\mathbf{D}, \mathbf{D}_{1}$ and $\mathbf{D}_{2}$, described in next subsection). For $\omega>\omega_{x}$, the system reenters the dynamically stable regime and exhibits again the previous behaviors, with an oscillatory resonant type evolution for $\omega$ above but close to $\omega_{x}$ (curve B in Fig. 2).

Close to instability but still within the stable regime, the maximum entanglement reached is of order $\ln \mid \omega-$ $\omega_{\mu} \mid:$ For $\omega$ close to $\omega_{\mu}(\mu=x, y)$ on the stable side, and for the initial conditions (28), $f(t)$ will be maximum at $t \approx t_{m}$, with

$$
f\left(t_{m}\right) \approx \frac{\omega \mid \varepsilon_{-}}{\lambda_{+}^{2} \lambda_{-}} \sqrt{\frac{\left(\frac{\alpha_{x} \alpha y+\omega^{2}}{\lambda_{+}}\right)^{2} \sin ^{2} \frac{m \pi \lambda_{+}}{2 \lambda_{-}}+\alpha_{\mu}^{2} \cos ^{2} \frac{m \pi \lambda_{+}}{2 \lambda_{-}}}{\alpha_{x} \alpha_{y}}},
$$

where $\lambda_{+} \approx \sqrt{2\left(\varepsilon_{+}+\omega_{\mu}^{2}\right)}$ and

$$
\lambda_{-} \approx \sqrt{\frac{2 \omega_{\mu}\left|\varepsilon_{-}\right|\left|\omega_{\mu}-\omega\right|}{\varepsilon_{+}+\omega_{\mu}^{2}}},
$$



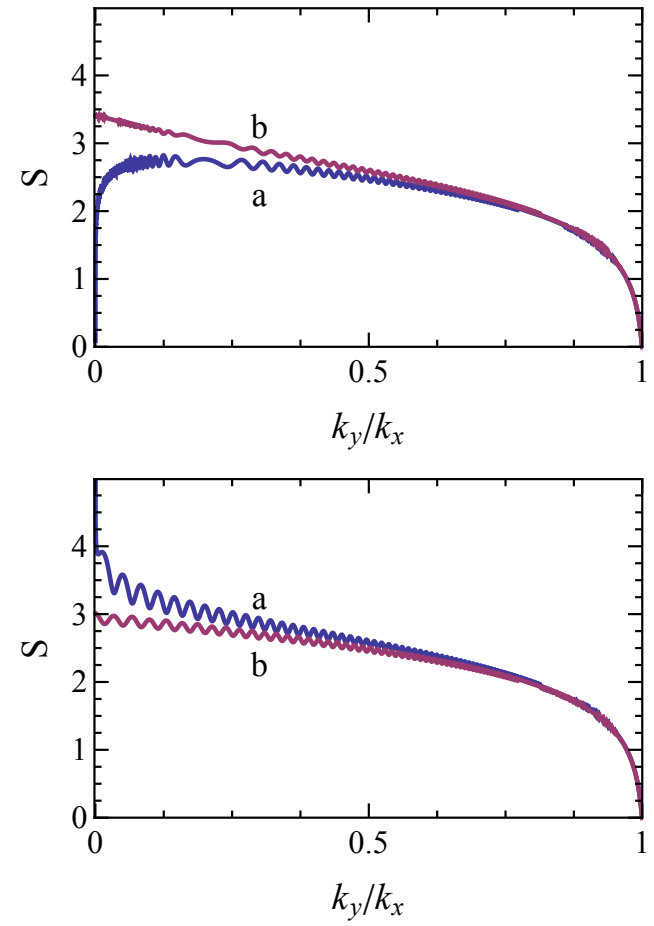

FIG. 3. The maximum entanglement $S\left(t_{m}\right)$ reached in stable sectors close to instability, as a function of the anisotropy ratio $k_{y} / k_{x}$ (see Eq. (32) ). Top: Vicinity of border A-D $(\omega=$ $\left.0.999 \omega_{y}\right)$. Bottom: Vicinity of border B-D $\left(\omega=1.001 \omega_{x}\right)$. The initial state is the separable ground state of $H_{0}\left(\alpha_{\mu}=\omega_{\mu}\right)$ in curves a and a separable isotropic state $\left(\alpha_{\mu}=1\right)$ in curves b.

implying $f\left(t_{m}\right)=O\left(\left|\omega_{\mu}-\omega\right|^{-1 / 2}\right)$ and hence $S\left(t_{m}\right)=$ $O\left(-\frac{1}{2} \ln \left|\omega_{\mu}-\omega\right|\right)$.

Expression (32) (and hence $S\left(t_{m}\right)$ ) will tend to decrease for decreasing anisotropy, i.e., increasing ratio $k_{y} / k_{x} \leq 1$, as seen in Fig. 3 for $m=1$, vanishing in the isotropic limit $k_{y} / k_{x} \rightarrow 1$ (where $f\left(t_{m}\right)=O\left(\mid k_{x}-\right.$ $\left.k_{y} \mid\right)^{1 / 2}$. On the other hand, the behavior for $k_{y} / k_{x} \rightarrow 0$ will depend on the initial condition: If it is the ground state of $H_{0}\left(\alpha_{\mu}=\omega_{\mu}\right.$, curves a), $f\left(t_{m}\right)$ will vanish at the first border $\omega \approx \omega_{y}$ (top panel), where $f\left(t_{m}\right)=O\left(\sqrt{\omega_{y}}\right)$, but diverge at the second border $\omega=\omega_{x}$ (bottom panel), where $f\left(t_{m}\right)=O\left(1 / \sqrt{\omega_{y}}\right)$, as obtained from Eq. (32). If the initial state is fixed, however, $f\left(t_{m}\right)$ will approach a finite value for $k_{y} / k_{x} \rightarrow 0$, and exhibit a monotonous decrease on average with increasing ratio $k_{y} / k_{x}$ in both borders (curves b in Fig. 31), as also implied by (32). We also mention that the high frequency oscillations in $f\left(t_{m}\right)$ and $S\left(t_{m}\right)$ observed in Fig. 3 stem from the $\lambda_{+} / \lambda_{-}$ratio in the arguments of the trigonometric functions in Eq. (32). For $\omega$ close to $\omega_{\mu}$, this ratio is minimum around $k_{y} / k_{x} \approx 1 / 5$, which leads to the observed decrease in the oscillation frequency of $S\left(t_{m}\right)$ in the vicinity of this ratio (top panel).

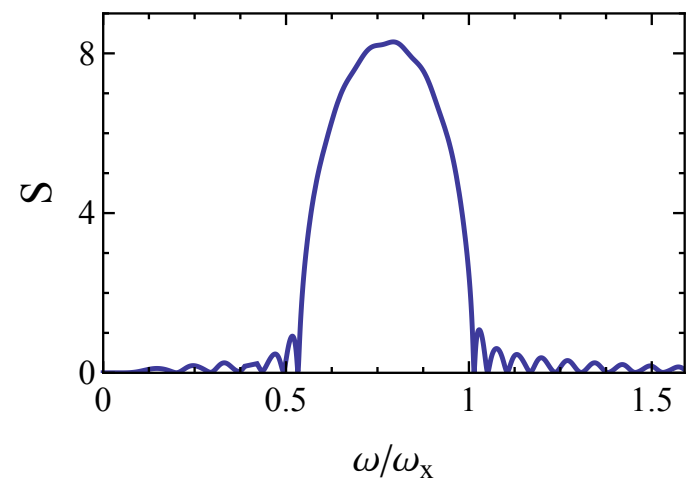

FIG. 4. The entanglement entropy between the two modes attained at fixed time $\omega_{x} t=40$, as a function of the (constant) frequency $\omega$, for the oscillator parameters and initial state of Fig. 2 Entanglement is bounded for $\omega<\omega_{y}$ (sector $\mathbf{A}$ ) and $\omega>\omega_{x}$ (sector $\mathbf{B}$ ), but is proportional to $t$ in the instability window $\omega_{y}<\omega<\omega_{x}$ (sector $\left.\mathbf{D}\right)$.

\section{Evolution in unstable sectors}

Let us now examine in detail the evolution of $S(t)$ in the dynamically unstable regimes. At the critical frequencies $\omega=\omega_{\mu}, \mu=y, x$ (borders A-D and B-D), $\lambda_{-}$ vanishes and Eqs. (12) and (24) lead, for large $t$ and the initial conditions (28), to the critical evolution

$$
f(t) \approx t \frac{\omega\left|\varepsilon_{-}\right|}{\lambda_{+}^{2}} \sqrt{\frac{\left(\frac{\alpha_{x} \alpha_{y}+\omega^{2}}{\lambda_{+}}\right)^{2} \sin ^{2} \lambda_{+} t+\alpha_{\mu}^{2} \cos ^{2} \lambda_{+} t}{\alpha_{x} \alpha_{y}}}
$$

where $\lambda_{+}=\sqrt{2\left(\varepsilon_{+}+\omega_{\mu}^{2}\right)}>0$. This entails a linear increase, on average, of $f(t)$ in this limit, and hence, a logarithmic growth of $S(t)$, according to Eq. (26):

$$
S(t) \approx S_{0}(t)+\ln t
$$

where $S_{0}(t)=1+\ln [f(t) / t]$ is a bounded function oscillating with frequency $\lambda_{+}$. This behavior (curve A-D in Fig. (2) is the $\omega \rightarrow \omega_{\mu}$ limit of the previous resonant regime.

On the other hand, in the unstable sector $\mathbf{D}\left(\omega_{y}<\omega<\right.$ $\left.\omega_{x}\right), \lambda_{-}$becomes imaginary. This leads to an exponential term in $f(t)\left(\frac{\sin \lambda_{-} t}{\lambda_{-}} \rightarrow \frac{\sinh \left|\lambda_{-}\right| t}{\left|\lambda_{-}\right|}\right)$, which will dominate the large $t$ evolution: In this sector Eqs. (12), (24) and (27) imply, for large $t$,

$$
f(t) \propto e^{\left|\lambda_{-}\right| t}, \quad S(t) \approx\left|\lambda_{-}\right| t,
$$

and hence, a linear growth (on average) of the entanglement entropy with time (curves $\mathbf{D}_{1}, \mathbf{D}_{2}$ in Fig. 2). Therefore, in the unstable window $\omega_{y} \leq \omega \leq \omega_{x}$, there is an unbounded growth with time of the entanglement entropy, which will originate a pronounced maximum in the generated entanglement at a given fixed time and anisotropy as a function of $\omega$, as appreciated in Fig. [- 


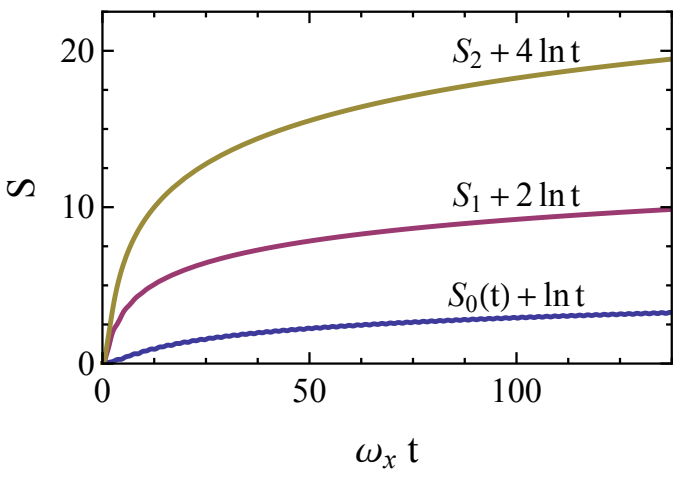

FIG. 5. Critical evolution of the entanglement entropy at the border between sectors with distinct dynamics, for isotropic initial conditions $\left(\alpha_{\mu}=1\right)$. The lower, middle and upper curve correspond respectively to the border $\mathbf{A}-\mathbf{D}$ (at $k_{y}=$ $0.5 k_{x}$, with $\omega=\sqrt{k_{y}}$ ), B-E (at $k_{y}=-1.5 k_{x}$, with $\omega$ given by (18) and the critical points $\mathbf{L}$ (Eq. 20). The asymptotic behavior for large $t$ (Eqs. 35, (38), 40) is indicated.

We now examine the behavior at the other sectors of Fig. [1. In the unstable sectors $\mathbf{C}$ and $\mathbf{E}$, where one or both of the constants $k_{\mu}$ are negative, $\lambda_{ \pm}$are imaginary or complex (Fig. 【). This implies an exponential increase of $f(t)$, as indicated in table प entailing again a linear asymptotic growth of the entanglement entropy with time: $S(t) \approx\left(\left|\lambda_{+}\right|+\left|\lambda_{-}\right|\right) t$ in $\mathbf{C}$ and $S(t) \approx 2\left|\operatorname{Im}\left(\lambda_{ \pm}\right)\right| t$ in $\mathbf{E}$, neglecting constant or bounded terms.

On the other hand, at the border between sectors $\mathbf{B}$ and $\mathbf{E}$, which corresponds to the critical curve $\Delta=0$ between both points $L$ in Fig. 1 we obtain, for large $t$ and $k_{x} \neq k_{y}$ (with the initial conditions (28)), the asymptotic behavior

$$
f(t) \approx\left|\varepsilon_{-}\right| \frac{4 \omega^{2} \alpha_{x} \alpha_{y}+\varepsilon_{-}^{2}}{16 \omega \lambda \sqrt{\alpha_{x} \alpha_{y}}} t^{2}
$$

where $\lambda=\sqrt{\varepsilon_{+}+\omega^{2}}>0$. This leads to

$$
S(t) \approx S_{1}+2 \ln t
$$

with $S_{1} \approx 1+\ln \left[\left|\varepsilon_{-}\right| \frac{4 \omega^{2} \alpha_{x} \alpha_{y}+\varepsilon_{-}^{2}}{16 \omega \lambda \sqrt{\alpha_{x} \alpha_{y}}}\right]$. Hence, the unbounded growth of $f(t)$ and $S(t)$ is here more rapid than that at the previous borders A-D and B-D $\left(\omega=\omega_{y}\right.$ or $\left.\omega_{x}\right)$ (quadratic instead of linear increase of $f(t)$ ). At the border E-C the asymptotic behavior of $f(t)$ is still exponential (i.e., linear growth of $S(t)$ ).

Finally, a further remarkable critical behavior arises at the special critical points $L$, obtained for condition (20), where all sectors $\mathbf{B}, \mathbf{C}, \mathbf{D}$ and $\mathbf{E}$ meet. We obtain here a purely polynomial evolution of $(f(t)+1 / 2)^{2}$, as implied by Eqs. 211. For large $t$, this leads to a quartic increase of $f(t)$ :

$$
f(t) \approx \frac{\alpha_{x} \alpha_{y}+\omega^{2}}{6 \sqrt{\alpha_{x} \alpha_{y}}} \omega^{3} t^{4}
$$

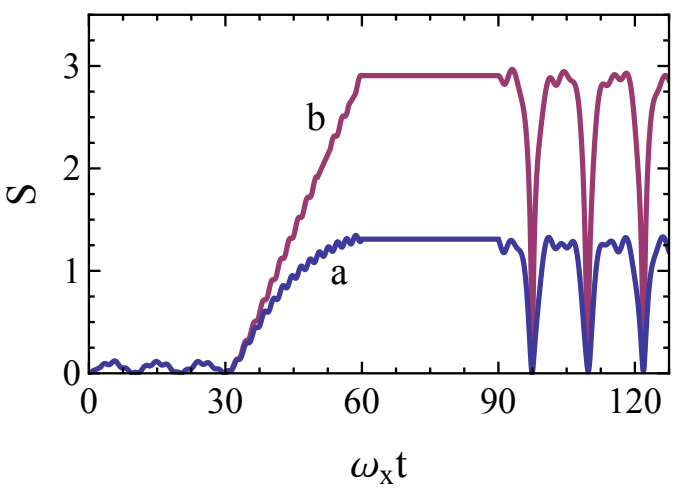

FIG. 6. Evolution of the entanglement entropy for a stepwise varying frequency $\omega$, starting from the separable ground state of $H_{0}$ (with $k_{y}=0.5 k_{x}>0$ ). In curve a we have set $\omega / \omega_{x}=$ $0.5,0.7,0$ and 0.21 for successive time intervals of length $\omega_{x} \Delta t=30$, such that the system is close to the first instability at the second interval $\left(0.7 \omega_{x} \approx 0.99 \omega_{y}\right.$, with $\left.\omega_{\mu}=\sqrt{k_{\mu}}\right)$, while in curve $\mathbf{b}$ the only change is $\omega=0.75 \omega_{x} \approx 1.06 \omega_{y}$ in the second interval, such that the system enters there the unstable regime leading to a linear entanglement growth. This plot shows that entanglement can be increased, kept constant and returned to a vanishing value just by tuning the frequency $\omega$.

implying the following logarithmic increase of $S(t)$ :

$$
S(t) \approx S_{2}+4 \ln t,
$$

where $S_{2} \approx 1+\ln \left[\frac{\alpha_{x} \alpha_{y}+\omega^{2}}{6 \sqrt{\alpha_{x} \alpha_{y}}} \omega^{3}\right]$. Hence, the increase is here still more rapid than at both previous borders. These critical behaviors are all depicted in Fig. 5

\section{Entanglement control}

We finally show in Fig. 6the possibilities offered by this model for controlling the entanglement growth through a stepwise time dependent frequency, starting from the separable ground state of $H_{0}$. After applying a "low" initial frequency $\omega=0.5 \omega_{x}$ for $\omega_{x} t<30$, which leads to a weak quasiperiodic entanglement, by tuning $\omega$ to a value close to the first instability $\omega_{y}=\sqrt{k_{y}}$ for a finite time $\left(30<\omega_{x} t<60\right)$, it is possible to achieve a large entanglement increase (curve a). Then, by setting $\omega=0$ (i.e., switching off the field or rotation), entanglement is kept high and constant, since the evolution operator becomes a product of local mode evolutions. Finally, by turning the frequency on again up to a low value, entanglement can be made to exhibit strong oscillations, practically vanishing at the minimum if $\omega$ is appropriately tuned. Thus, disentanglement at specific times can be achieved if desired. The entanglement increase at the second interval can be enhanced by allowing the system to enter the instability region for a short time, as shown in curve b. 


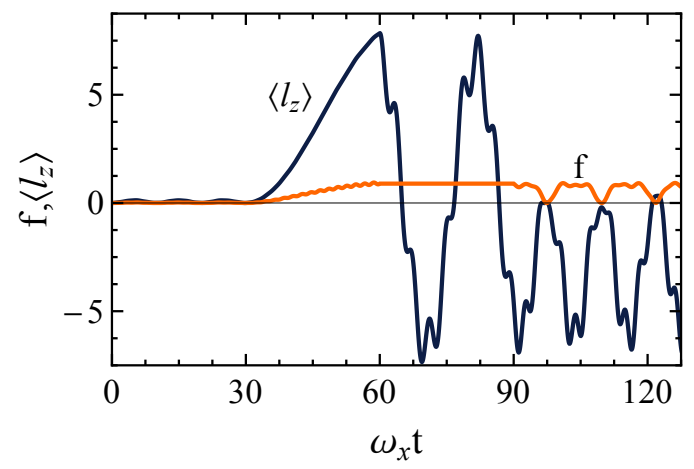

FIG. 7. Evolution of the average occupation number $f(t)$ (Eq. (24) ) and angular momentum $\left\langle l_{z}\right\rangle$ for case a of Fig. 6.

The growth of the average occupation $f(t)$ (and hence the entanglement entropy $S(t)$ ) in the second interval is strongly correlated with that of the average angular momentum $\left\langle l_{z}\right\rangle_{t}$, i.e., with the entangling term in $H$, as seen in Fig. 7 for case a of Fig. [6. Nonetheless, while the evolution of $f(t)$ is similar to $S(t)$, the average angular momentum exhibits pronounced oscillations when $\omega$ is switched off, since $l_{z}$ is not preserved in the present anisotropic trap $\left(\left[l_{z}, h_{0}\right] \neq 0\right)$. These oscillations persist in the last interval, although shifted and partly attenuated. Here the vanishing of $\left\langle l_{z}\right\rangle_{t}$ provides a check for the vanishing entanglement, since in a separable state $\left\langle l_{z}\right\rangle=\left\langle q_{x}\right\rangle\left\langle p_{y}\right\rangle-\left\langle q_{y}\right\rangle\left\langle p_{x}\right\rangle$ and for the present initial conditions $\left\langle q_{\mu}\right\rangle=\left\langle p_{\mu}\right\rangle=0$ for all times. Thus, $f(t)=0$ implies here $\left\langle l_{z}\right\rangle_{t}=0$, although the converse is not valid.

Though initially correlated, we remark that $\left\langle l_{z}\right\rangle_{t}$ and the average occupation $f(t)$ do not have a fixed asymptotic relation in the whole plane. For instance, at the stability borders $\omega=\omega_{\mu},\left\langle l_{z}\right\rangle_{t}$ increases on average as $t^{2}$ for high $t$, i.e., as $f^{2}(t)$ (Eq. (34)), and the same relation with $f(t)$ holds in the unstable sector $\mathbf{D}\left(\omega_{y}<\omega<\omega_{x}\right)$, where $\left\langle l_{z}\right\rangle_{t} \propto e^{2\left|\lambda_{-}\right| t}$. Nonetheless, in an unstable potential at the critical curve $\Delta=0,\left\langle l_{z}\right\rangle_{t} \propto t^{2}$ (on average) for large $t$, increasing then as $f(t)$ (Eq. (37)), while at the critical points $\mathbf{L}$ we obtain $\left\langle l_{z}\right\rangle_{t} \propto t^{6}$, i.e., $\left\langle l_{z}\right\rangle_{t} \propto f^{3 / 2}(t)$ asymptotically (Eq. (39)).

\section{CONCLUSIONS}

We have analyzed the entanglement generated by an angular momentum coupling between two harmonic modes, when starting from a separable gaussian state. The general treatment considered here is fully analytic and valid throughout the entire parameter space, including stable and unstable regimes, as well as critical regimes where the system cannot be written in terms of normal coordinates or independent quadratic systems (non-diagonalizable $\mathcal{H}^{2}$ ). Hence, in spite of its simplicity, the present model is able to exhibit different types of en- tanglement evolution, including quasiperiodic evolution, linear growth, and also logarithmic growth of the entanglement entropy with time, which can all be reached just by tuning the frequency. The model is then able to mimic the typical evolution regimes of the entanglement entropy encountered in more complex many-body systems. Even distinct types of critical logarithmic growth can be reached when allowing for general quadratic potentials. The system offers then the possibility of an easily controllable entanglement generation and growth, through stepwise frequency changes, which can also be tuned in order to disentangle the system at specific times. The model can therefore be of interest for continuous variable based quantum information.

The authors acknowledge support from CONICET (LR,NC) and CIC (RR) of Argentina. We also thank Prof. S. Mandal for motivating discussions during his visit to our institute.

\section{A. Appendix}

In order to highlight the non-trivial character of the present model when considered for all real values of the constants $k_{\mu}$ and frequency $\omega>0$, we provide here some further details 20]. With the sole exception of the critical curve $\Delta=0$ (Eq. (18)), the Hamiltonian (3) can be written as a sum of two quadratic Hamiltonians,

$$
h=\frac{1}{2}\left(\alpha_{+} p_{+}^{2}+\beta_{+} q_{+}^{2}\right)+\frac{1}{2}\left(\alpha_{-} p_{-}^{2}+\beta_{-} q_{-}^{2}\right),
$$

where $p_{ \pm}, q_{ \pm}$are related with $q_{x, y}, p_{x, y}$ by the linear canonical transformation

$$
\begin{aligned}
q_{ \pm} & =\frac{q_{x, y}-\eta p_{y, x}}{(1+\gamma \eta)}, \quad p_{ \pm} & =p_{x, y}+\gamma q_{y, x} \\
\gamma & =\left(\Delta-\varepsilon_{-}\right) /(2 \omega), \quad \eta & =\gamma / \varepsilon_{+}
\end{aligned}
$$

such that $\left[q_{r}, p_{s}\right]=i \delta_{r s},\left[q_{r}, q_{s}\right]=\left[p_{r}, p_{s}\right]=0$ for $r, s=$ \pm , and

$$
\alpha_{ \pm}=\frac{1}{2}+\frac{\varepsilon_{-} \pm 2 \omega^{2}}{2 \Delta}, \quad \beta_{ \pm}=\frac{\Delta}{\omega^{2}}\left(\Delta \alpha_{ \pm}-\varepsilon_{-}\right),
$$

with $\alpha_{ \pm} \beta_{ \pm}=\lambda_{ \pm}^{2}$ (Eq. (10)). Nonetheless, the coefficients $\alpha_{ \pm}, \beta_{ \pm}$can be positive, negative or zero, and may become even complex, according to the values of $k_{x}, k_{y}$ and $\omega$. We may obviously interchange $p_{ \pm}$with $q_{ \pm}$in (42) by a trivial canonical transformation $p_{ \pm} \rightarrow q_{ \pm}, q_{ \pm} \rightarrow-p_{ \pm}$. This freedom in the final form will be used in the following discussion.

In sector $\mathbf{A}$ of Fig. 1 $\alpha_{ \pm}, \beta_{ \pm}$in Eq. (41) are both real and positive, and the system is equivalent to two harmonic modes. Here $\lambda_{ \pm}$are both real. In sector $\mathbf{B}$, $\alpha_{+}, \beta_{+}$are positive but $\alpha_{-}, \beta_{-}$are both negative, so that the system is here equivalent to a standard plus an "inverted" oscillator. Nevertheless, $\lambda_{ \pm}$remain still real. In sector $\mathbf{C}, \alpha_{ \pm} \beta_{ \pm}<0$, and the effective quadratic potential becomes unstable in both coordinates (i.e., $\alpha_{ \pm}>0$, $\left.\beta_{ \pm}<0\right)$. Here $\lambda_{ \pm}$are both imaginary. In sector $\mathbf{D}, \alpha_{+}$ 
and $\beta_{+}$are positive but $\alpha_{-} \beta_{-}<0$, so that the potential is stable in one direction but unstable in the other (i.e., $\left.\alpha_{-}>0, \beta_{-}<0\right)$. Here $\lambda_{+}$is real but $\lambda_{-}$is imaginary. In sector $\mathbf{E}$, both $\alpha_{ \pm}$and $\beta_{ \pm}$are complex and $q_{ \pm}, p_{ \pm}$are non hermitian [20]. Here $\lambda_{ \pm}$are complex conjugates.

At the border $\mathbf{A}-\mathbf{D}, \alpha_{+}, \beta_{+}, \alpha_{-}$are all positive but $\beta_{-}=0$ (or similar with $\beta_{-} \leftrightarrow \alpha_{-}$), so that the system is equivalent to a harmonic oscillator plus a free particle $\left(\lambda_{+}>0, \lambda_{-}=0\right)$. The same holds at the border B-D, except that $\alpha_{-}<0$ (inverted free particle term). At the Landau point $\mathbf{F}\left(k_{x}=k_{y}=\omega^{2}\right.$, where $\mathbf{A}, \mathbf{B}$, and $\mathbf{D}$ meet), $\alpha_{+}>0, \beta_{+}>0$ but $\alpha_{-}=\beta_{-}=0$. Finally, at the border $\mathbf{C}-\mathbf{D}, \alpha_{+}>0, \beta_{+}=0$ (or similar with $\beta_{+} \leftrightarrow \alpha_{+}$) and $\alpha_{-} \beta_{-}<0$, implying $\lambda_{+}=0$ and $\lambda_{-}$imaginary.
The decomposition (41) no longer holds at the critical curve $\Delta=0$, which separates sector $\mathbf{E}$ from sectors $\mathbf{C}$ and D. At this curve (including points $\mathbf{L}$ ), the system is inseparable, in the sense that it cannot be written as a sum of two independent quadratic systems, even if allowing for complex coordinates and momenta as in sector E. While the matrix $\mathcal{H}^{2}$ (Eq. (9)) is always diagonalizable for $\Delta \neq 0$, i.e., whenever the decomposition (41) is feasible, both $\mathcal{H}$ and $\mathcal{H}^{2}$ are non-diagonalizable when $\Delta=0$. Here $\lambda_{ \pm}=\lambda$, with $\lambda$ real at the border between $\mathbf{B}$ and $\mathbf{E}$, imaginary between $\mathbf{C}$ and $\mathbf{E}$ and zero at the points $\mathbf{L}$. The optimum decomposition of $h$ in these cases is discussed in 20].
[1] J. Schachenmayer, B.P. Lanyon, C.F. Roos, A.J. Daley, Phys. Rev. X 3031015 (2013).

[2] J.H. Bardarson, F. Pollmann, J.E. Moore, Phys. Rev. Lett. 109017202 (2012).

[3] A.J. Daley, H. Pichler, J. Schachenmayer, P. Zoller, Phys. Rev. Lett. 109020505 (2012).

[4] C.H. Bennett et al., Phys. Rev. Lett. 70, 1895 (1993); Phys. Rev. Lett. 76, 722 (1996).

[5] M.A. Nielsen and I.L. Chuang, Quantum Computation and Quantum Information (Cambridge Univ. Press, Cambridge, UK, 2000).

[6] R. Josza and N. Linden, Proc. R. Soc. A 459, 2011 (2003).

[7] G. Vidal, Phys. Rev. Lett. 91, 147902 (2003).

[8] N. Schuch, M. M.Wolf, F. Verstraete, and J. I. Cirac, Phys. Rev. Lett. 100, 030504 (2008).

[9] J.G. Valatin, Proc. R. Soc. London 238, 132 (1956).

[10] A. Feldman and A. H. Kahn, Phys. Rev. B 1, 4584 (1970).

[11] P. Ring and P. Schuck, The Nuclear Many-Body Problem, (Springer, NY, 1980).

[12] J.P. Blaizot and G. Ripka, Quantum Theory of Finite Systems (MIT Press, MA, 1986).

[13] A.V. Madhav, T. Chakraborty, Phys. Rev. B 49, 8163 (1994).

[14] M. Linn, M. Niemeyer, and A. L. Fetter, Phys. Rev. A 64, 023602 (2001).

[15] M. Ö. Oktel, Phys. Rev. A 69, 023618 (2004).

[16] A. L. Fetter, Phys. Rev. A 75, 013620 (2007).

[17] A. Aftalion, X. Blanc, and N. Lerner, Phys. Rev. A 79, 011603(R) (2009).

[18] A. Aftalion, X. Blanc, J. Dalibard, Phys. Rev. A 71, 023611 (2005); S. Stock et al, Laser Phys. Lett. 2, 275 (2005).

[19] A.E. Fetter, Rev. Mod. Phys. 81, 647 (2009). I. Bloch, J. Dalibard, W. Zwerger, Rev. Mod. Phys. 80, 885 (2008);

[20] R. Rossignoli and A.M. Kowalski, Phys. Rev. A 79
062103 (2009).

[21] R.F. Werner, M.M. Wolf, Phys. Rev. Lett. 86, 3658 (2001).

[22] K. Audenaert, J. Eisert, M.B. Plenio, and R.F. Werner, Phys. Rev. A 66, 042327 (2002).

[23] G. Adesso, A. Serafini, and F. Illuminati, Phys. Rev. A 70, 022318 (2004); A. Serafini, G. Adesso, and F. Illuminati, Phys. Rev. A 71, 032349 (2005).

[24] S.L. Braunstein and P. van Loock, Rev. Mod. Phys. 77, 513 (2005).

[25] C. Weedbrook et al, Rev. Mod. Phys. 84, 621.

[26] J. Pěrina, Z. Hradil, and B. Jurčo, Quantum optics and Fundamentals of Physics (Kluwer, Dordrecht, 1994); N. Korolkova, J. Pěrina, Opt. Comm. 136, 135 (1996).

[27] L. Rebón, R. Rossignoli, Phys. Rev. A 84, 052320 (2011).

[28] A.P. Hines, R. H. McKenzie, and G.J. Milburn, Phys. Rev. A 67, 013609 (2003).

[29] H.T. Ng, P.T. Leung, Phys. Rev. A 71, 013601 (2005).

[30] A.V. Chizhov, R.G. Nazmitdinov, Phys. Rev. A 78, 064302 (2008).

[31] V. Sudhir, M.G. Genoni, J. Lee, M.S. Kim, Phys. Rev. A 86, 012316 (2012).

[32] R. Rossignoli and C.T. Schmiegelow, Phys. Rev. A 75, 012320 (2007).

[33] L. Amico, et al, Phys. Rev. A bf 69, 022304 (2004); A. Sen(De), U. Sen, and M. Lewenstein, Phys. Rev. A 70, 060304(R) (2004); 72, 052319 (2005).

[34] S.D. Hamieh and M.I. Katsnelson, Phys. Rev. A 72, 032316 (2005); Z. Huang, S. Kais, Phys. Rev. A 73, 022339 (2006); M. Koniorczyk, P. Rapcan, V. Buzek, Phys. Rev. A 72, 022321 (2005).

[35] L. Amico, R. Fazio, A. Osterloh and V. Vedral, Rev. Mod. Phys. 80, 516 (2008).

[36] A.K. Ekert et al, Phys. Rev. Let.. 88, 217901 (2002); C.M. Alves, D. Jaksch, Phys. Rev. Let.. 93, 110501 (2004). 